\title{
Crystal structures of the TRIC trimeric intracellular cation channel orthologues
}

\author{
Go Kasuya ${ }^{1}$, Masahiro Hiraizumi ${ }^{1}$, Andrés D Maturana ${ }^{2}$, Kaoru Kumazaki ${ }^{1}$, Yuichiro Fujiwara ${ }^{3}$, \\ Keihong Liu ${ }^{4}$, Yoshiko Nakada-Nakura ${ }^{4}$, So Iwata ${ }^{4}$, Keisuke Tsukada ${ }^{1}$, Tomotaka Komori ${ }^{1}$, Sotaro Uemura ${ }^{1}$, \\ Yuhei Goto ${ }^{5}$, Takanori Nakane ${ }^{1}$, Mizuki Takemoto ${ }^{1}$, Hideaki E Kato ${ }^{1,10}$, Keitaro Yamashita ${ }^{6}$, Miki Wada ${ }^{7}$, \\ ${\text { Koichi } \text { Ito }^{8} \text {, Ryuichiro Ishitani }}^{1}$, Motoyuki Hattori ${ }^{9}$, Osamu Nureki ${ }^{1}$
}

${ }^{I}$ Department of Biological Sciences, Graduate School of Science, The University of Tokyo, 2-11-16 Yayoi, Bunkyo-ku, Tokyo 1130032, Japan; ${ }^{2}$ Department of Bioengineering Sciences, Graduate School of Bioagricultural Sciences, Nagoya University, Furo-cho, Chikusa-ku, Nagoya 464-8601, Japan; ${ }^{3}$ Integrative Physiology, Department of Physiology, Graduate School of Medicine, Osaka University, 2-2 Yamada-oka, Suita, Osaka 565-0871, Japan; ${ }^{4}$ Department of Cell Biology, Graduate School of Medicine, Kyoto University, Yoshida-Konoe-cho, Sakyo-ku, Kyoto 606-8501, Japan; ${ }^{5}$ Laboratory of Chromosome Dynamics, Institute of Molecular and Cellular Biosciences, The University of Tokyo, 1-1-1, Yayoi, Bunkyo-ku, Tokyo 113-0032, Japan; ${ }^{6}$ SR Life Science Instrumentation Unit, RIKEN SPring-8 Center, 1-1-1 Kouto, Sayo-cho, Sayo-gun, Hyogo 679-5148, Japan; ${ }^{7}$ Technical office, The Institute of Medical Science, The University of Tokyo, 4-6-1 Shirokanedai, Minato-ku, Tokyo 108-8639, Japan; ${ }^{8}$ Department of Medical Genome Sciences, Graduate School of Frontier Sciences, The University of Tokyo, 5-1-5 Kashiwanoha, Kashiwa-shi, Chiba 277-8562, Japan; ' State Key Laboratory of Genetic Engineering, Collaborative Innovation Center of Genetics and Development, Department of Physiology and Biophysics, School of Life Sciences, Fudan University, 2005 Songhu Road, Shanghai 200438, China

$\mathrm{Ca}^{2+}$ release from the sarcoplasmic reticulum (SR) and endoplasmic reticulum (ER) is crucial for muscle contraction, cell growth, apoptosis, learning and memory. The trimeric intracellular cation (TRIC) channels were recently identified as cation channels balancing the $\mathrm{SR}$ and ER membrane potentials, and are implicated in $\mathrm{Ca}^{2+}$ signaling and homeostasis. Here we present the crystal structures of prokaryotic TRIC channels in the closed state and structure-based functional analyses of prokaryotic and eukaryotic TRIC channels. Each trimer subunit consists of seven transmembrane (TM) helices with two inverted repeated regions. The electrophysiological, biochemical and biophysical analyses revealed that TRIC channels possess an ion-conducting pore within each subunit, and that the trimer formation contributes to the stability of the protein. The symmetrically related TM2 and TM5 helices are kinked at the conserved glycine clusters, and these kinks are important for the channel activity. Furthermore, the kinks of the TM2 and TM5 helices generate lateral fenestrations at each subunit interface. Unexpectedly, these lateral fenestrations are occupied with lipid molecules. This study provides the structural and functional framework for the molecular mechanism of this ion channel superfamily.

Keywords: $\mathrm{Ca}^{2+}$ homeostasis; ion channels; X-ray crystallography; electrophysiology

Cell Research (2016) 26:1288-1301. doi:10.1038/cr.2016.140; published online 2 December 2016

\section{Introduction}

Correspondence: Motoyuki Hattori ${ }^{\mathrm{a}}$, Osamu Nureki ${ }^{\mathrm{b}}$

${ }^{\mathrm{a} E}$ E-mail: hattorim@fudan.edu.cn

bE-mail: nureki@bs.s.u-tokyo.ac.jp

${ }^{10}$ Current address: Department of Molecular and Cellular Physiology, Stanford University School of Medicine, Stanford, California 94305, USA.

Received 8 October 2016; revised 23 October 2016; accepted 27 October 2016; published online 2 December 2016
$\mathrm{Ca}^{2+}$ release from the sarcoplasmic reticulum (SR) and endoplasmic reticulum (ER) is involved in various cellular functions, including muscle contraction, cell growth, apoptosis, learning and memory [1-4]. $\mathrm{Ca}^{2+}$-releasing channels, including ryanodine receptor (RyR) and inositol trisphosphate receptor (IP3R), as well as $\mathrm{Ca}^{2+}$ uptake pumps, are essential components for this process, and their molecular mechanisms have been functionally and structurally characterized [5-11]. $\mathrm{Ca}^{2+}$ release and uptake 
further necessitate counter-monovalent cation movements to balance the membrane potentials of the SR and ER, because $\mathrm{Ca}^{2+}$ release from and uptake into the SR and ER generate negative and positive potentials, respectively, inside the SR and ER, which attenuate the processes of $\mathrm{Ca}^{2+}$ release and uptake [12-14]. However, the molecular mechanism that balances the SR and ER membrane potentials for $\mathrm{Ca}^{2+}$ release and uptake has remained enigmatic.

The TRIC family proteins are trimeric intracellular cation channels localized at the SR and ER [15]. They were identified as the counter-cation channels that facilitate and maintain $\mathrm{Ca}^{2+}$ release from the SR and ER [15]. In addition to their function as counter-cation channels $\left(\mathrm{K}^{+}\right.$ export) upon $\mathrm{Ca}^{2+}$ release, a recent electrophysiological study suggested that TRIC channels are also important for maintaining the $\mathrm{K}^{+}$balance across the resting SR via $\mathrm{K}^{+}$uptake [16]. A recent bioinformatics analysis predicted the existence of prokaryotic homologues, with both the eukaryotic and prokaryotic homologues commonly possessing seven transmembrane (TM) helices per subunit [17]. In higher eukaryotes, such as human and mouse, two different TRIC subtypes, TRIC-A and TRIC-B, were identified and functionally characterized [18-21]. TRIC-A and TRIC-B both function as monovalent cation channels, with a weak preference for $\mathrm{K}^{+}$ions $[22,23]$. TRIC-A is predominantly expressed in excitable tissues, including muscle, while TRIC-B is widely expressed in various tissues. The tric-a-knockout mice showed compromised RyR-mediated $\mathrm{Ca}^{2+}$ release in vascular smooth muscle cells [24-26]. As tric- $a$ is associated with hypertension risk [24], TRIC-A is recognized as a potential drug target for malignant hypertension [19]. In contrast, tric- $b$ knockout mice exhibited abnormal IP3R-mediated $\mathrm{Ca}^{2+}$ release at the ER in airway epithelial cells [27], as well as compromised collagen production and impaired bone mineralization [28]. Mutations in tric- $b$ genes are responsible for osteogenesis imperfecta [29-31]. Together, these findings indicated that TRIC-mediated $\mathrm{K}^{+}$permeation plays an important role in $\mathrm{Ca}^{2+}$ signaling and homeostasis in the SR and ER. Despite their physiological importance, the lack of structural information about the TRIC proteins has hindered the elucidation of the molecular mechanism of cation conduction by TRIC channels.

Here we report the crystal structures of prokaryotic TRIC channels, together with the functional analyses of prokaryotic and eukaryotic TRIC channels, providing the molecular basis for the function of this superfamily.

\section{Results}

Functional characterization and structure determination of prokaryotic TRIC proteins
To understand the molecular mechanism of cation permeation by TRIC channels, we conducted functional and structural analyses of the prokaryotic TRIC homologues. We first screened for the expression of prokaryotic TRIC proteins by fluorescence-detection size-exclusion chromatography (FSEC) and FSEC-based thermostability assays [32, 33], and identified two prokaryotic TRIC proteins as suitable candidates for structural studies: a bacterial TRIC protein, RsTRIC from Rhodobacter sphaeroides ( $R$. sphaeroides), and an archaeal TRIC protein, SsTRIC from Sulfolobus solfataricus (S. solfataricus) (Figure 1A).

To functionally characterize the prokaryotic TRIC proteins, we employed an in vivo complementation assay and a patch-clamp analysis. The moderate expression of either RsTRIC or SsTRIC in the $\mathrm{K}^{+}$-requiring Escherichia coli (E. coli) strain, a derivative of the TK $405 \mathrm{~m}$ strain [34], successfully complemented the $\mathrm{K}^{+}$auxotroph in low-KCl medium, in contrast to the control empty vector (Figure 1B). We performed the patch-clamp analysis of giant spheroplasts of E. coli expressing RsTRIC, and successfully measured the $\mathrm{K}^{+}$currents associated with RsTRIC (Figure 1C and 1E). The giant spheroplasts harboring the empty vector did not exhibit this activity (Figure 1D). We were unable to measure the currents associated with SsTRIC, presumably due to the functional incompatibility of the archaeal SsTRIC with the E. coli giant spheroplast membrane, but the details remain unknown. Accordingly, we mainly employed the RsTRIC for the functional analyses of prokaryotic TRIC channels. Overall, these results demonstrate that the prokaryotic TRIC proteins possess ion channel activity.

The initial crystals of the prokaryotic TRIC proteins were obtained by the vapor diffusion method, but diffracted X-rays poorly. The combination of a C-terminal truncation and detergent screening yielded the crystals of RsTRIC and SsTRIC, which diffracted X-rays to $\sim 3.5$ $\AA$ and $\sim 6 \AA$ resolutions, respectively. The C-terminal truncations of RsTRIC and SsTRIC (RsTRIC $\triangle \mathrm{C} 8$ and SsTRIC $\Delta$ C7) did not affect the ability to rescue bacterial growth under $\mathrm{K}^{+}$auxotrophic condition (Figure 1B). The initial structure of RsTRIC was then solved by single-wavelength anomalous diffraction using the selenomethionine-labeled protein, and was further refined to $3.4 \AA$ resolution using the native data set (Supplementary information, Figure S1C, S1D and Table S1). Further crystallization of SsTRIC in complex with the Fab fragment of a monoclonal antibody against SsTRIC yielded crystals diffracting up to $2.6 \AA$ resolution. We then determined the SsTRIC-Fab complex structure by molecular replacement, using the RsTRIC and Fab structures as search models (Supplementary information, Figure S1A, 
A

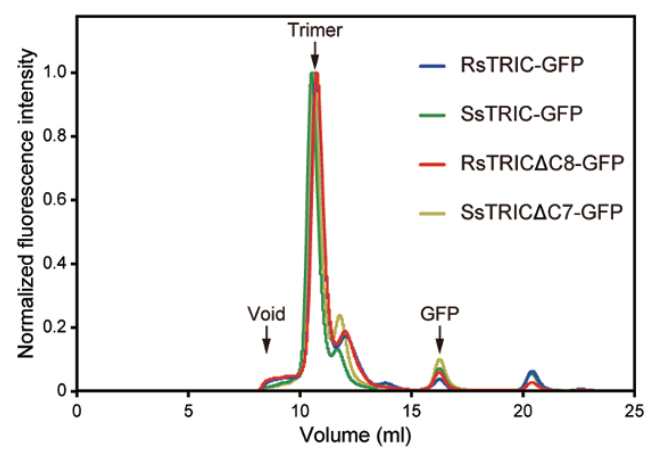

C

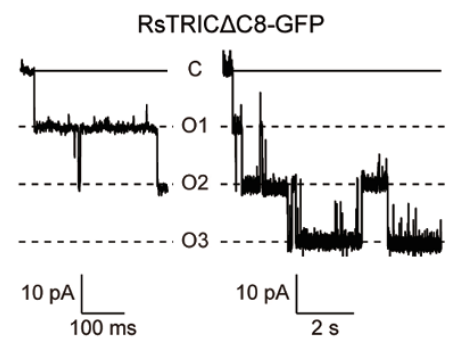

B

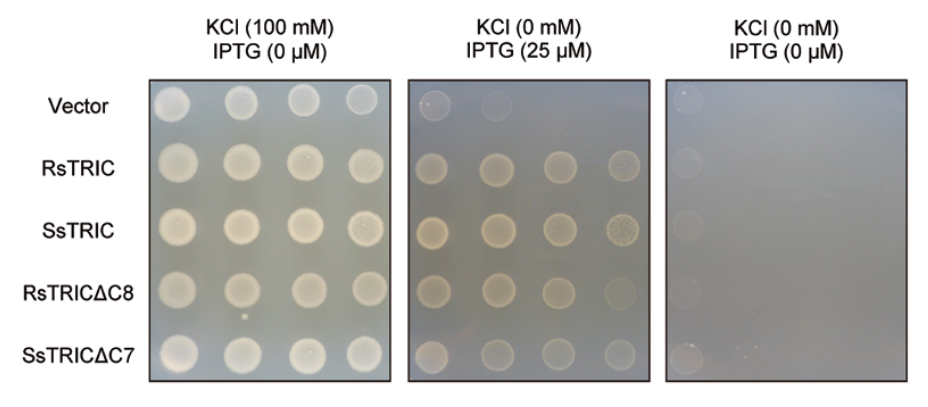

D

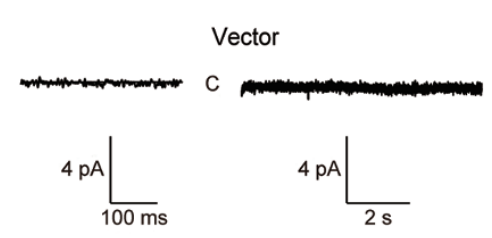

E

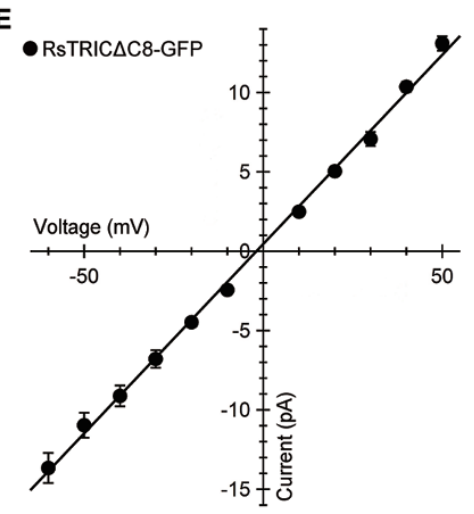

Figure 1 Functional characterization of prokaryotic TRIC proteins. (A) FSEC profiles for GFP-tagged RsTRIC (blue), SsTRIC (green), RsTRIC $\Delta$ C8 (red) and SsTRIC $\Delta$ C7 (yellow). The arrows indicate the elution positions of the void volume (void), the trimer of TRIC-GFP (trimer) and the free GFP (GFP). (B) Growth complementation assay of $\mathrm{K}^{+}$auxotrophic strains harboring either the empty vector or the expression vectors encoding GFP-tagged prokaryotic TRIC proteins. A 10-fold dilution series was spotted on low-potassium medium plates. (C, D) Representative current traces recorded at $-60 \mathrm{mV}$ in a membrane patch of $E$. coli giant spheroplasts expressing GFP-tagged RsTRIC $\Delta \mathrm{C} 8(n=6)(\mathrm{C})$, or harboring the empty vector $(n=3)(\mathrm{D})$ in $\mathrm{KCl}$-containing bath medium. In each panel, an enlarged trace is presented on the left. Uppercase letters, C and O1-O3, indicate closed and open states, respectively. Currents were recorded for $9 \mathrm{~s}$ in each step-pulse, and the single channel was observed as a triple event. (E) Current-voltage relationship ( $I-V$ curve), determined by measuring the current amplitude from $-60 \mathrm{mV}$ to $+40 \mathrm{mV}$ by $10 \mathrm{mV}$ steps. The values presented represent mean $\pm \operatorname{SEM}(n=6)$.

\section{S1B and Table S1).}

\section{Overall structure of the SsTRIC and RsTRIC trimers}

The crystallographic asymmetric unit of the SsTRICFab complex contains three SsTRIC subunits and three Fab molecules (Supplementary information, Figure S1E). The N- and C-terminal ends of SsTRIC extend into the periplasm and cytoplasm, respectively, according to the positive-inside rule [35] and the previous topological analysis of eukaryotic TRIC proteins [15]. The Fab molecules are bound to the periplasmic surface (Supplementary information, Figures S1F and S1G). The membrane orientation of SsTRIC and RsTRIC is also supported by the result that both proteins were expressed as the C-terminally GFP-tagged proteins, since GFP does not properly fold on the periplasmic side of E. coli [36]. SsTRIC forms a trimer, with each subunit related by non-crystallographic threefold symmetry (Figure 2A and Supplementary information, Figure S1E). Similarly, RsTRIC also forms a trimer. The asymmetric unit of RsTRIC contains two subunits, and each subunit is related by crystallographic threefold symmetry (Figure 2B and Supplementary information, Figure S1H). Therefore, the prokaryotic TRIC proteins form trimers, consistent with the previous chemical cross-linking and electron microscopy analyses of mouse TRIC-A [15].

The RsTRIC subunit and trimer are both quite similar to those of SsTRIC, with root mean square deviation (RMSD) values below $1.4 \AA$ for $188 \mathrm{C} \alpha$ atoms of the subunits and $2.3 \AA$ for $549 \mathrm{C} \alpha$ atoms of the trimers (Sup- 
A
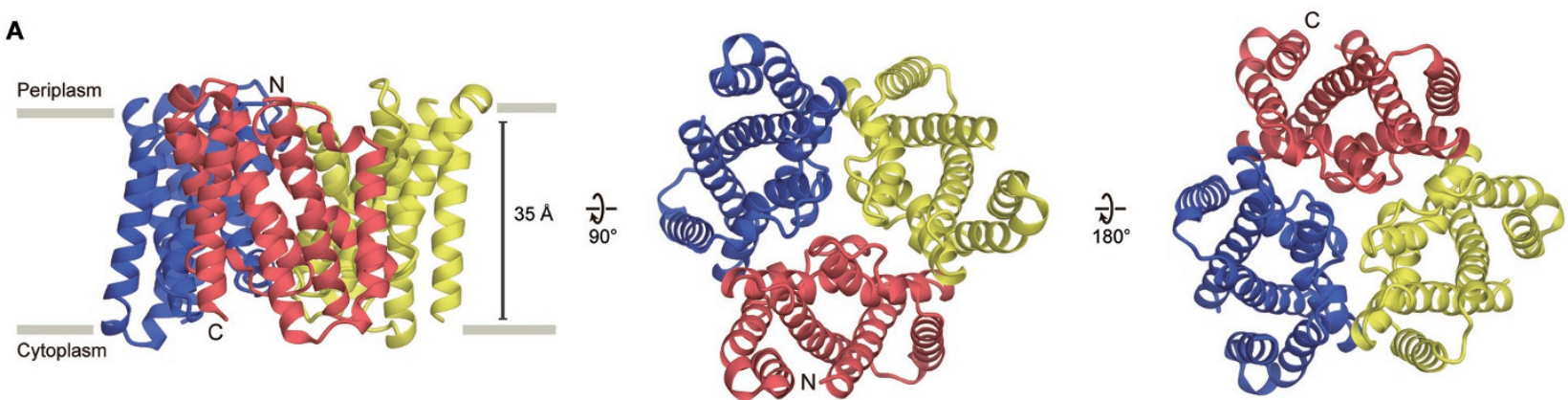

B
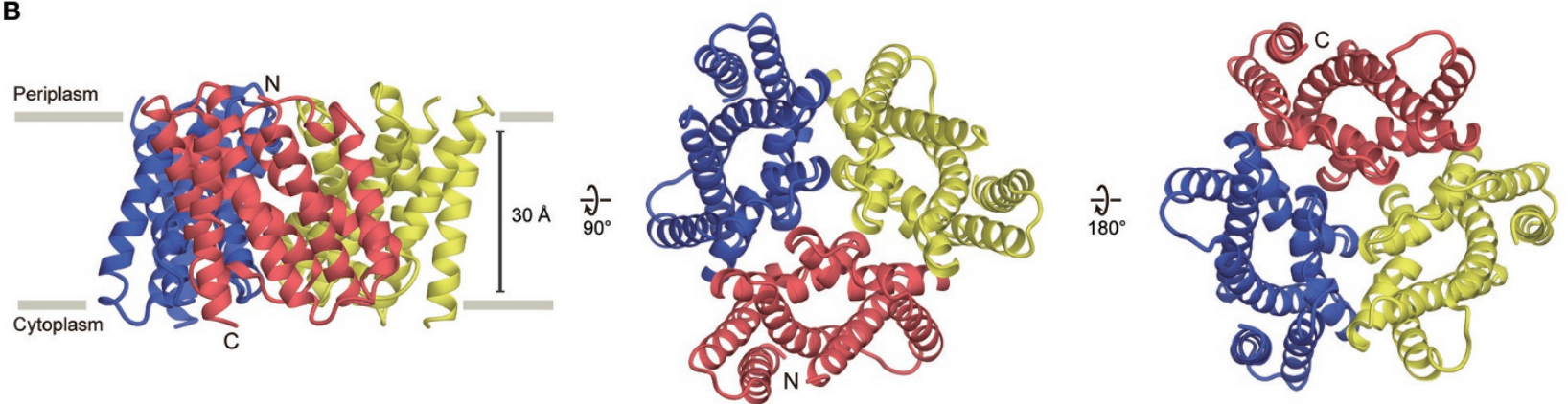

C
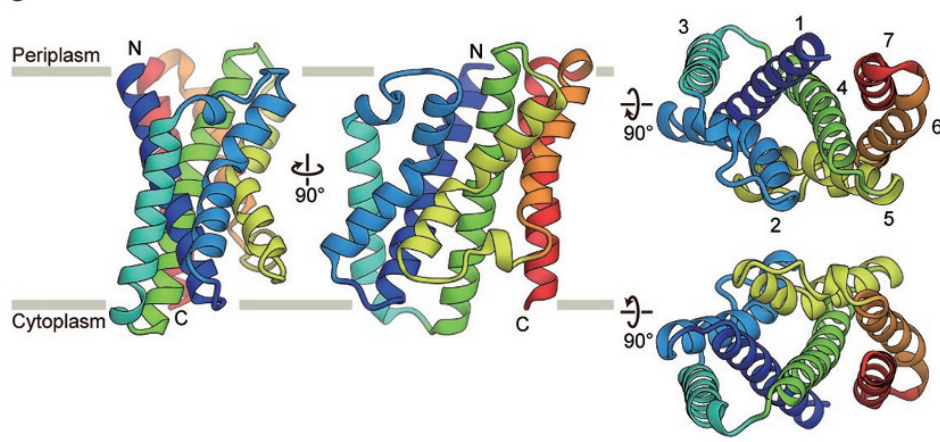

D

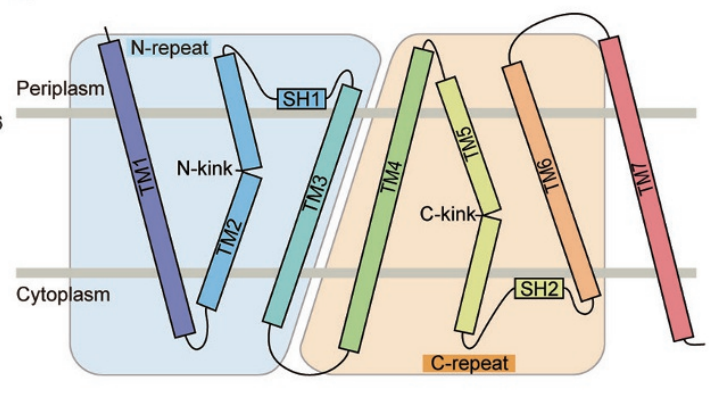

E

F
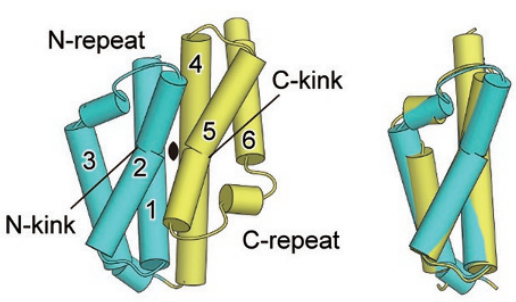

G
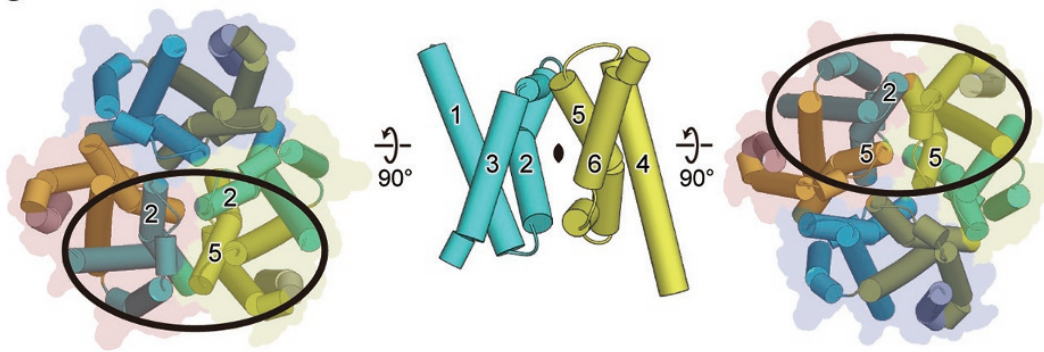

Figure 2 Overall structures and subunit folds of the SsTRIC and RsTRIC trimers. (A, B) Overall structures of the SsTRIC (A) and RsTRIC (B) trimers, viewed parallel to the membrane (left), and from the periplasmic (middle) and cytoplasmic (right) sides. Each subunit is colored differently (red, blue and yellow). (C) Structure of the SsTRIC subunit, viewed parallel to the membrane (left), and from the periplasmic and cytoplasmic sides (right), colored blue to red from the $N$ to $C$ terminus. (D) Schematic representation of the SsTRIC membrane topology, colored as in C. (E) Cartoon representation of the SsTRIC subunit, viewed parallel to the membrane. The $\mathrm{N}$-repeat is colored cyan and the C-repeat is colored yellow. TM7 was removed to show the twofold symmetry. (F) Superimposition of the N-repeat and the C-repeat. (G) The twofold symmetry relationship between the $\mathrm{N}$-repeat and the C-repeat of the other subunit, colored as in $\mathbf{E}$, viewed from the periplasmic side (left), parallel to the membrane (middle), and from the cytoplasmic side (right). 
plementary information, Figure S2). Since the structure of SsTRIC was solved at higher resolution, we mainly describe the SsTRIC structure unless noted otherwise.

\section{Subunit architecture and trimer interface}

Each SsTRIC subunit is composed of seven TM helices (Figure 2C and 2D), and consists of two repeated regions (Figure 2E) arisen by gene duplication, as indicated by the previous bioinformatics analysis [17]. The first three TM helices (N-repeat) are homologous to the second three TM helices (C-repeat), with the RMSD value of $1.5 \AA$ for $81 \mathrm{C} \alpha$ atoms (Figure $2 \mathrm{~F}$ ). The N- and C-repeats are related by the pseudo-twofold symmetry axis passing through the subunit center, parallel to the lipid bilayer (Figure 2E). Accordingly, these two repeated regions are arranged antiparallel to each other, with tight interactions between the TM1 and TM2 helices in the N-repeat and the TM4 and TM5 helices in the C-repeat (Figure 2E). The TM2 helix in the N-repeat and the corresponding TM5 helix in the C-repeat are kinked at the glycine residues (N-kink: Gly45 and Gly46 in TM2, C-kink: Gly130, Gly132, Gly133 and Gly134 in TM5) (Figure 2D, 2E and Supplementary information, Figure S3A). These glycine residues are highly conserved among the eukaryotic and prokaryotic TRIC proteins, despite the weak overall sequence identity of the prokaryotic TRIC proteins with the eukaryotic homologues (Supplementary information, Figure S3).

The intra-subunit pseudo-twofold symmetry of each subunit yields a pseudo dimer of trimers, with symmetrical inter-subunit interactions on the periplasmic and cytoplasmic sides (Figure 2G). On the periplasmic side, the TM2 and following SH1 helices in the N-repeat of one subunit mainly interact with both the TM2 and TM5 helices in the neighboring subunit (Figure 2G). Furthermore, hydrophobic interactions exist between the side chains of Ile51 in the TM2 helices at the center of the trimer (Supplementary information, Figure S4A). Likewise, extensive interactions are formed on the cytoplasmic side between the TM5 helix in the C-repeat of one subunit and the TM2 and TM5 helices in the neighboring subunit (Figure 2G), and the side chains of Val139 in the TM5 helices from the subunits face each other at the cytoplasmic center of the trimer (Supplementary information, Figure S4A). Taken together, these symmetrical inter-subunit interactions mediated by the TM2 and TM5 helices contribute to the trimer formation of the TRIC channels.

\section{Monomeric pore of TRIC channels}

Inspections of the TRIC structures suggested two possible pathways for ion conduction. One pathway is the "monomeric pore" at the center of each subunit (Figure 4A-4D). The TRIC channels possess a solvent accessible cavity on the periplasmic side in each subunit, and this putative pathway is closed on the cytoplasmic side (Figure 4A and 4C). The other pathway is the "trimeric pore". The TRIC structures revealed the presence of a putative ion-conducting pore at the center of the trimer, formed by the symmetrically arranged TM2 and TM5 helices (Supplementary information, Figure S4C and S4D). In our structures, these "trimeric pores" are closed by hydrophobic residues on both the periplasmic and cytoplasmic sides (Supplementary information, Figure S4A and S4B).

To determine whether the TRIC channels possess the ion-conducting pore within each subunit or at the center of the trimer, we first designed and created a series of mutants to monomerize RsTRIC. We mutated the residues at the trimer interface to bulky hydrophobic residues (Supplementary information, Figure S4A-S4D), and found that one of these mutants (L52W/I134W/L138W/ L $139 \mathrm{~W} / \mathrm{I} 153 \mathrm{~F}$ ) exhibited no trimer peak by FSEC screening (Figure 3A). We then assessed the oligomeric state of this mutant in detergent-soluble (Figure 3B) and E. coli cell membrane-associated (Figure 3C) forms by chemical cross-linking experiments using a cross-linker, disuccinimidyl suberate (DSS). While the treatment with increasing amounts of DSS produced the band corresponding to the covalent trimer of RsTRIC, we did not observe the corresponding band of this mutant (Figure $3 \mathrm{~B}$ and $3 \mathrm{C}$ ), indicating the absence of trimer formation by the mutant in both soluble and membrane-associated forms. To further investigate the properties of this mutant in the membrane, we conducted the single-molecule fluorescence analysis with zero-mode waveguides (ZMWs) [37] (Figure 3D-3F). ZMWs are photonic nano-structures that provide a highly confined observation volume [37-39], allowing the detection of a single fluorescent molecule even at relatively high concentrations. We employed the ZMWs for the single-molecule fluorescence photobleaching assay. RsTRIC channels with a C-terminal HaloTag were labeled with a fluorophore, tetramethyl rhodamine (TMR), and incorporated into nanodiscs composed of lipid bilayers from $E$. coli lipids with membrane scaffold proteins (MSPs). In this experiment, the number of channel subunits can be estimated by counting the number of photobleaching steps. The representative ZMW traces and the statistical analysis for RsTRIC showed one, two and three photobleaching steps, indicating the trimer formation by RsTRIC (Figure 3D-3F). In contrast, those for the mutant primarily showed one photobleaching step, but not three photobleaching steps, indicating that the mutant did not form a trimer (Figure 
3D-3F). Taken together, our series of biochemical and biophysical experiments in detergent, E. coli cell membrane and nanodiscs indicate that this mutant exists as a monomer, rather than a trimer.

To examine the functionality of this "monomeric" mutant, we performed the patch-clamp analysis. The $E$. coli spheroplasts expressing this mutant still exhibited the ion channel activity (Figure 3G-3I). We then assessed the stability of this mutant by the FSEC-based thermostability assay (FSEC-TS) [33]. In FSEC-TS, the monomerizing mutations had a drastic effect on the stability of RsTRIC. The calculated melting temperature $\left(T_{\mathrm{m}}\right)$ of the monomeric mutant was decreased by $24.7^{\circ} \mathrm{C}$ as compared with that of the wild type (Figure $3 \mathrm{~J}$ ), indicating that the trimer formation may contribute to the stability of the protein.
A

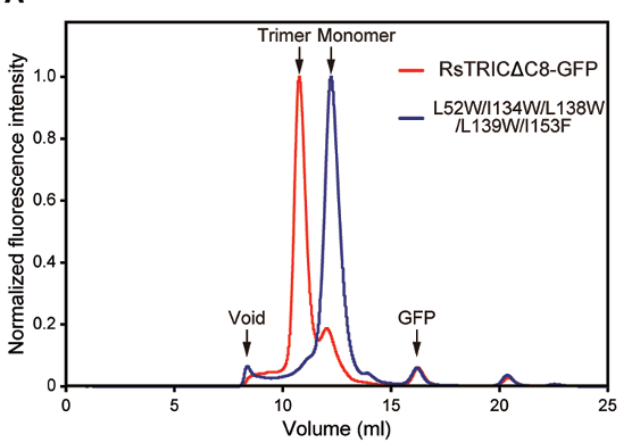

D

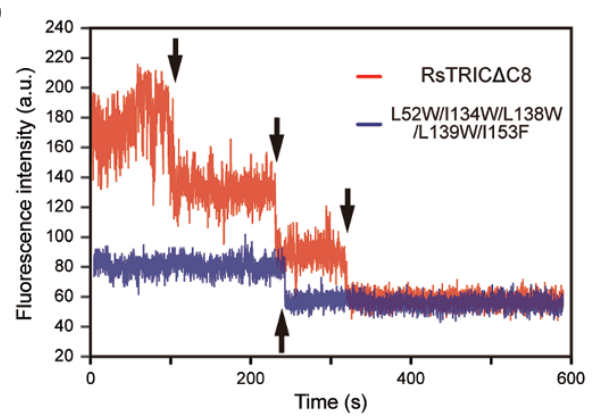

L52W/134W/L138W/L139W/153F (monomer)

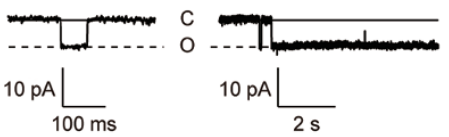

J

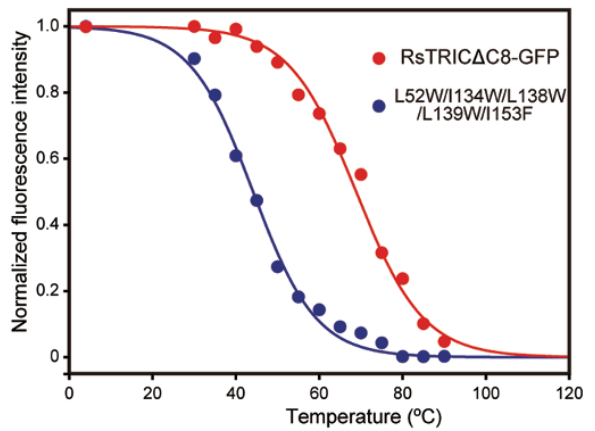

B RsTRIC $\Delta$ C8-GFP L52W/134W/L138W DSS $(\mu \mathrm{M}) \quad$ DSS $(\mu \mathrm{M})$ - $202002000-2002000$

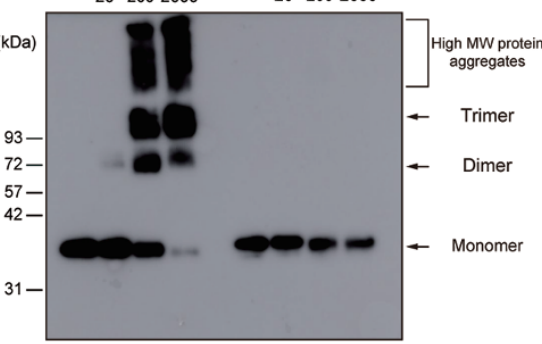

E

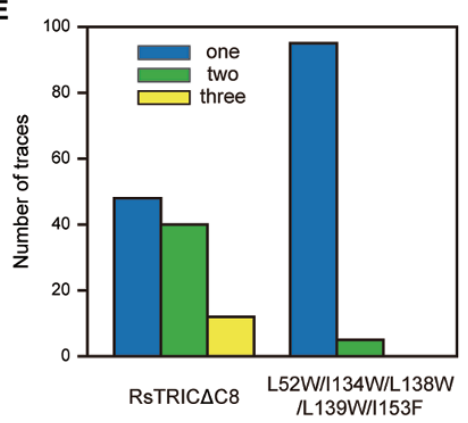

H

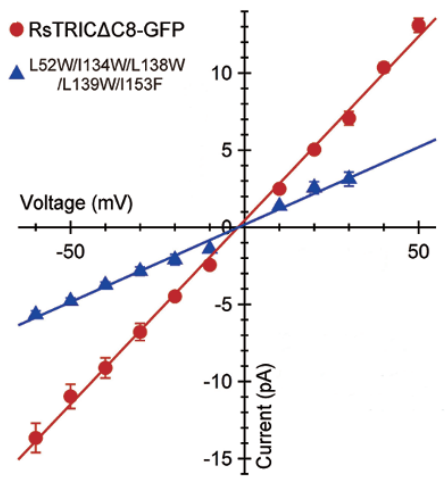

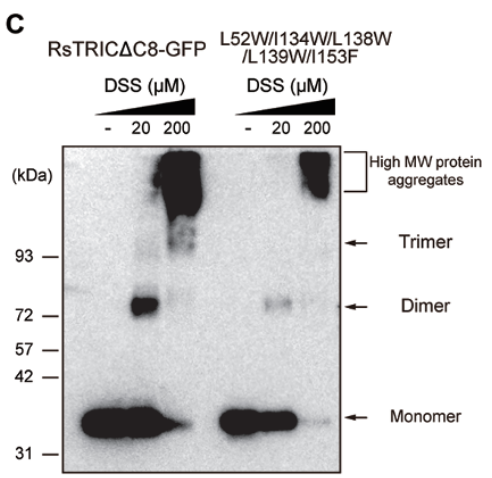

F

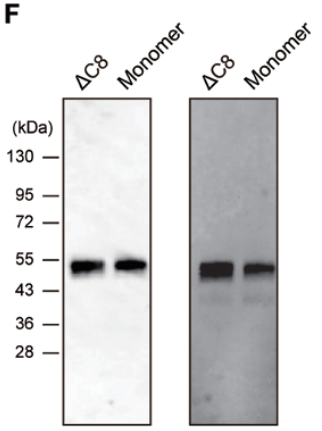


Taken together, our results demonstrate that RsTRIC possesses an ion-conducting pathway within each subunit. The trimer formation is not requisite for the gating activity, but contributes to the stability of the protein.

\section{Conserved glycine clusters in the putative channel pore}

The putative monomeric pore of the TRIC channels is mainly formed by the TM1, TM2, TM4 and TM5 helices (Figure 4A-4D), and is solvent-accessible on the periplasmic side. In contrast, this putative pathway is closed on the cytoplasmic side (Figure 4A and 4C). Accordingly, the current structures represent the closed state of the channel. On the cytoplasmic side, extensive hydrogen bonds exist between the main-chain carbonyl atom of Ala94 and the side chains of Asp97, Arg137, Tyr153 and Arg 187 (Supplementary information, Figure S4E). These residues are highly conserved among prokaryotes (Supplementary information, Figure S3A), and may contribute to the closed conformation in the current structure, implying the possible disruption of these hydrogen bonds upon channel opening.

The center of the putative monomeric pore consists of the glycine clusters (N-kink in TM2 and C-kink in TM5) and the two in-line phenylalanine residues, which close the pore on the periplasmic side with their bulky hydrophobic side chains (Phe16 and Phe104 in SsTRIC, and Phe17 and Phe103 in RsTRIC) in the TM1 and TM4 helices (Figure 4A-4D). These glycine clusters are highly conserved in both eukaryotes and prokaryotes (Supplementary information, Figure S3A), whereas these phenylalanine residues are only conserved among the pro- karyotic orthologues (Supplementary information, Figure S3A).

To investigate the functional role of the glycine clusters in the TM2 and TM5 regions and the two in-line phenylalanine residues in the TM1 and TM4 regions, we constructed the RsTRIC mutants of the N-kink (G45A/ G47A), C-kink (G132A/G133A) and two phenylalanines (F17A and F103A). The F17A and F103A mutants of RsTRIC were not expressed in E. coli, possibly suggesting the importance of the interactions mediated by these phenylalanine residues for the structural integrity, but further mutational analyses would be required to understand the importance of these residues in channel gating. The N- and C-kink mutants of RsTRIC were both expressed, but they exhibited no activity in the single-channel recording using the $E$. coli giant spheroplasts (Figure 4E, 4F and Supplementary information, Figure S5). These results suggest that the glycine clusters in the TM2 and TM5 regions are important for the ion channel activity. Considering the inverted pseudo-twofold symmetry of the TRIC channel (Figure 2E and 2F), it is tempting to speculate that the kink motions of the TM2 and TM5 helices at the conserved glycine clusters may cause the expansion of the TM domain to open the channel.

\section{Functional characterization of eukaryotic TRIC channels}

To examine the functional and mechanistic similarity between the eukaryotic and prokaryotic TRIC channels, we established an assay for eukaryotic TRIC channel function (Supplementary information, Figure S6). The yeast strain lacking the potassium/sodium exporter

Figure 3 Monomeric mutant of RsTRIC. (A) FSEC profiles on a Superdex 200 Increase 10/300 GL column (GE Healthcare) for the GFP-tagged RsTRIC $\Delta$ C8 (red) and monomeric mutant (blue). The arrows indicate the elution positions of the void volume (void), the trimer of TRIC-GFP (trimer), the monomer of TRIC-GFP (monomer) and the free GFP (GFP). (B, C) Cross-linking experiments with the GFP-tagged RsTRIC $\Delta$ C8 and monomeric (L52W/L134W/L138W/L139W/I153F) mutant in detergent-soluble $(\mathbf{B})$ and $E$. coli cell membrane-associated (C) forms. The theoretical molecular weight of the GFP-tagged RsTRIC $\Delta$ C8 monomer is about $40.5 \mathrm{kD}$. Increasing amounts of DSS (0,20, 200 and $2000 \mu \mathrm{M}$ for soluble samples, and 0 , 20 and $200 \mu \mathrm{M}$ for membrane-associated samples) were used to cross-link the proteins. The brackets and arrows indicate high-molecular-weight (high MW) protein aggregates, and the trimer, dimer and monomer, respectively. (D) Representative ZMW traces of TMR-labeled RsTRIC $\Delta$ C8 (red) and monomeric mutant (blue) in nanodiscs. The black arrows represent photobleaching steps. (E) Event histograms for TMR-labeled RsTRIC $\Delta$ C8 $(n=100)$ and the monomeric mutant $(n=100)$. The TMR-labeled RsTRIC $\Delta$ C8 showed 48, 40 and 12 events for one, two and three photobleaching steps, and the monomeric mutant showed 95 and 5 events for one and two photobleaching steps, respectively. (F) Detection of HaloTag-fused RsTRIC proteins by an anti-His antibody western blot (left) and TMR fluorescence (right). In each panel, " $\Delta$ C8" and "Monomer" indicate the TMR-labeled RsTRIC $\Delta$ C8 and monomeric mutant, respectively. (G) Representative current traces recorded at -60 $\mathrm{mV}$ in a membrane patch of $E$. coli giant spheroplasts expressing the GFP-tagged monomeric mutant in $\mathrm{KCl}$-containing solution $(n=5)$. Currents were recorded for $9 \mathrm{~s}$ in each step-pulse. An enlarged trace is presented on the left. Uppercase letters, $\mathrm{C}$ and $\mathrm{O}$, indicate closed and open states, respectively. $(\mathrm{H})$ Comparison of the current-voltage relationships $(I-V$ curve $)$ in the GFP-tagged RsTRIC $\Delta$ C8 (red) and the monomeric mutant (blue), determined by measuring the current amplitude from -60 $\mathrm{mV}$ to $+40 \mathrm{mV}$ by $10 \mathrm{mV}$ steps. The values are means \pm SEM ( $n=6$ in GFP-tagged RsTRIC $\Delta \mathrm{C} 8 ; n=5$ in monomeric mutant). (I) The open probabilities of the GFP-tagged RsTRIC $\Delta$ C 8 and monomeric mutant at $-60 \mathrm{mV}$. Bars depict means + SEM $(n=6$ in GFP-tagged RsTRIC $\Delta \mathrm{C} 8 ; n=5$ in monomeric mutant). (J) The melting curves of RsTRCl $\Delta \mathrm{C} 8\left(\mathrm{red}, T_{\mathrm{m}}=68.8^{\circ} \mathrm{C}\right)$ and the monomeric mutant (blue, $T_{\mathrm{m}}=44.1^{\circ} \mathrm{C}$ ). 
A

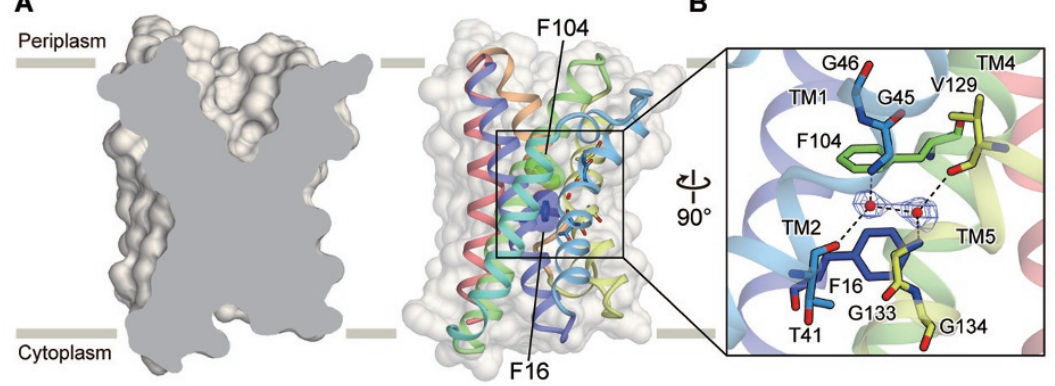

C

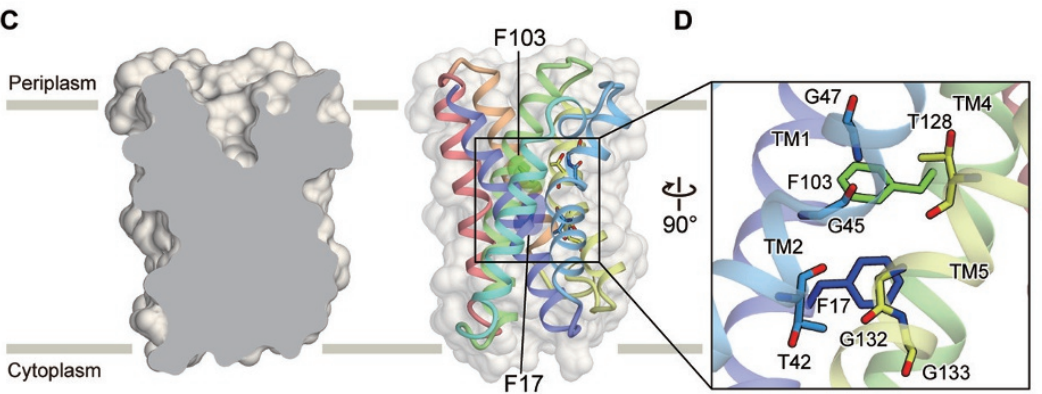

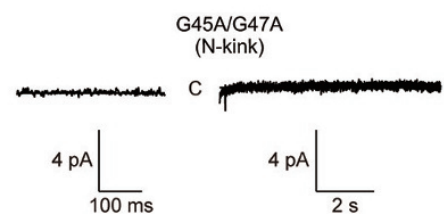

$\mathbf{F}$

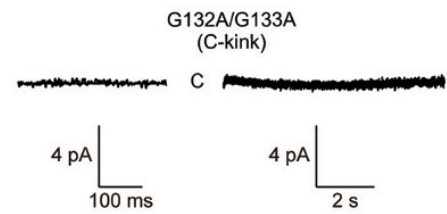

Figure 4 Putative ion channel pore. (A, C) Cross-sections of surface representations of the SsTRIC (A) and RsTRIC (C) subunits, showing the ion channel pores. The Phe residues at the constriction sites are depicted by spheres. (B, D) Close-up views of the centers of the pores in SsTRIC (B) and RsTRIC (D). Water molecules are depicted by red spheres. The $F_{\circ}-F_{c}$ electron density maps for water molecules, contoured at $3.5 \sigma$, are shown. (E, F) Representative current traces recorded at $-60 \mathrm{mV}$ in a membrane patch of spheroplasts expressing the GFP-tagged RsTRIC $\Delta \mathrm{C} 8 \mathrm{~N}$-kink (G45A/G47A; $n=3)(\mathrm{E})$ and C-kink (G132A/G133A; $n=3)(F)$ mutants. In each trace, an enlarged trace is presented on the left. The uppercase $C$ indicates the closed state.

genes, nha and enal-ena4, the tandemly arranged ena1 homologous gene cluster, exhibits growth sensitivity under high $\mathrm{KCl}$ conditions [40], and we constructed a similar strain to test the potassium uptake activity of eukaryotic TRIC channels. Caenorhabditis elegans TRIC1 (ceTRIC-1) was fused to the signal peptide of yeast alpha-mating factor at the N-terminus, and to the EGFP tag followed by the ER export signal (to target the ceTRIC-1 expression at the cell membrane [41], because TRICs are intracellular channels localized at SR and $\mathrm{ER})$ at the C-terminus. We confirmed that the overexpressed ceTRIC-1 was indeed localized at the yeast cell surface (Supplementary information, Figure S6A and $\mathrm{S} 6 \mathrm{~B}$ ), and increased the growth sensitivity under high $\mathrm{KCl}$ conditions, indicating that eukaryotic TRIC channels can uptake $\mathrm{K}^{+}$, as we also observed in the $E$. coli complementary assay with prokaryotic TRIC channels (Figure 1B). We further applied this assay to conduct the structure-based mutational analysis of eukaryotic TRICs (Supplementary information, Figure S6A). We created the C-kink (G163A/G165A/G167A) mutant of ceTRIC-1 (Supplementary information, Figure S6A and S6C).
This mutant was overexpressed and localized at the cell surface in yeast (Supplementary information, Figure S6B), but its overexpression had a much smaller effect on the growth sensitivity compared with the wild type (Supplementary information, Figure S6A). These results further indicate the mechanistic similarity between the eukaryotic and prokaryotic TRIC channels, in which the conserved glycine clusters are important for the channel activity.

\section{Lateral fenestration occupied with lipids}

The trimeric structures of SsTRIC and RsTRIC further revealed an unexpected feature: three lateral "fenestrations" at each of the subunit interfaces, which provide access from the membrane to the center of the trimeric channel (Figure 5A-5C and Supplementary information, Figure S7A-S7C). Each of these lateral fenestrations is about $20 \AA$ long and highly hydrophobic (Figure 5B-5E and Supplementary information, Figure S7B-S7E). The lateral fenestrations are mainly formed by the TM2 helix from one subunit and the TM5 helix from the neighboring subunit at the subunit interface related by the pseudo 
twofold symmetry (Figure 2G, 5A-5C and Supplementary information, Figure S7A-S7C).

We observed elongated electron densities within these lateral fenestrations (Figure 5B, 5C and Supplementary information, Figure S7B and $\mathrm{S} 7 \mathrm{C}$ ). Considering the length of the observed elongated electron densities and the hydrophobic environment of the lateral fenestrations, lipid molecules may occupy the lateral fenestrations in lipid bilayers. The acyl chains of the lipid molecules would extensively interact with the hydrophobic residues on the TM2 and TM5 helices at the lateral fenestrations (Figure 5D, 5E and Supplementary information, Figure S7D and S7E). Consistently, the molecular dynamics (MD) simulations of the trimeric SsTRIC channel embedded in a POPC membrane showed that the hydrophobic tails of the POPC molecules slid into the lateral fenestrations in the first $25 \mathrm{~ns}$, and remained there throughout the simulation (Supplementary information, Figure S7F and S7G). Although it is difficult to conclude whether these acyl chains in the current TRIC structures are derived from lipids or detergents, the MD simulation indicated that the acyl chains of lipid molecules could stably occupy the lateral fenestrations. Intriguingly, similar lateral fenestrations and cavities with accommodated lipid molecules were also observed in prokaryotic voltage-sensing $\mathrm{Na}^{+}$channel structures and mechanosensitive two-pore domain $\mathrm{K}^{+}$channel structures [42-44], and are implicated in the modulation of their gating.

\section{Structural comparisons of prokaryotic and eukaryotic} TRIC channels

Yang et al. [45] reported the crystal structures of eukaryotic TRIC-B channels from Caenorhabditis elegans (CeTRIC-B1 in a $\mathrm{Ca}^{2+}$-bound, potentially "activated", closed state and CeTRIC-B2 in a $\mathrm{Ca}^{2+}$-free, potentially "pre-activated", closed state) just before the submission of this manuscript. Since the CeTRIC-B1 and CeTRIC-B2 structures are similar, we employed
A
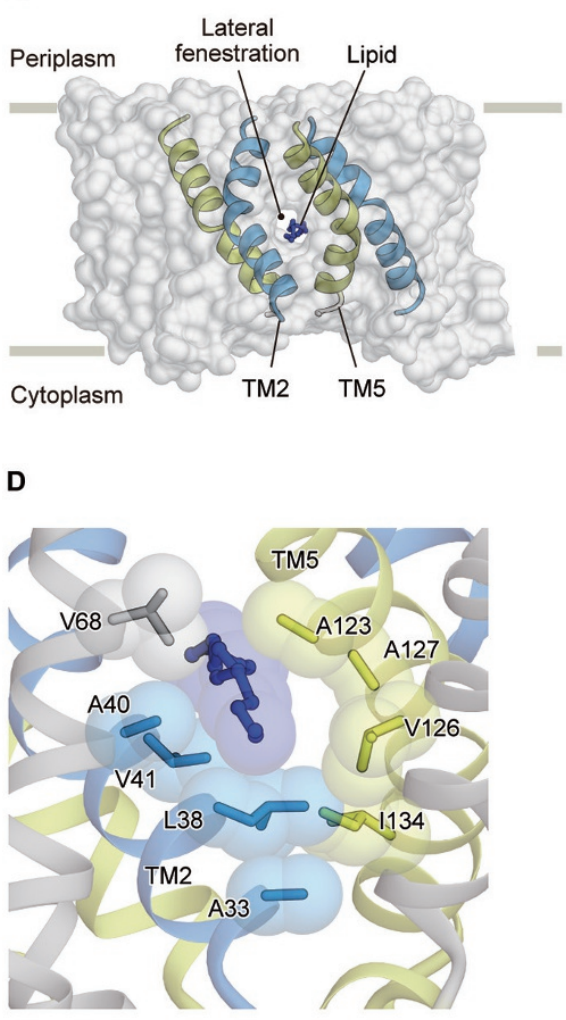

B

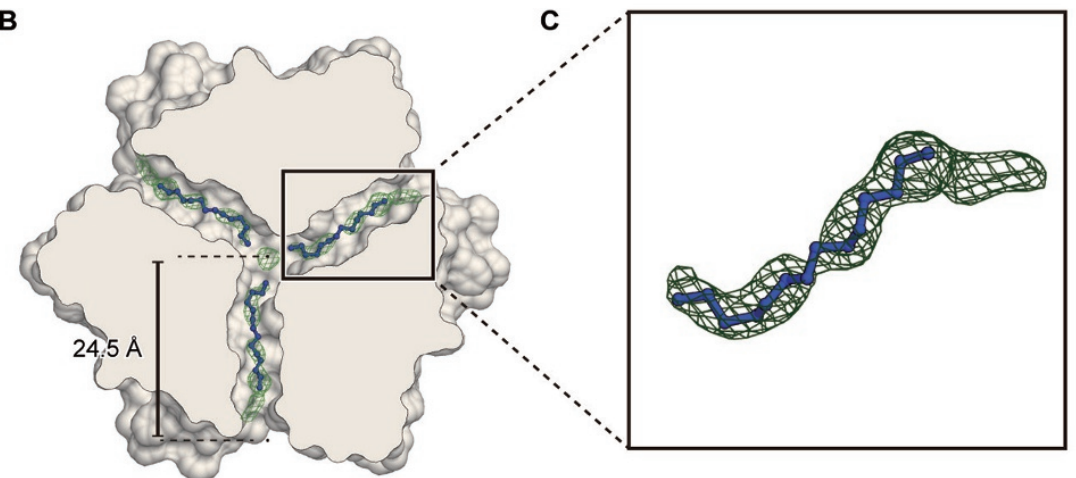

E

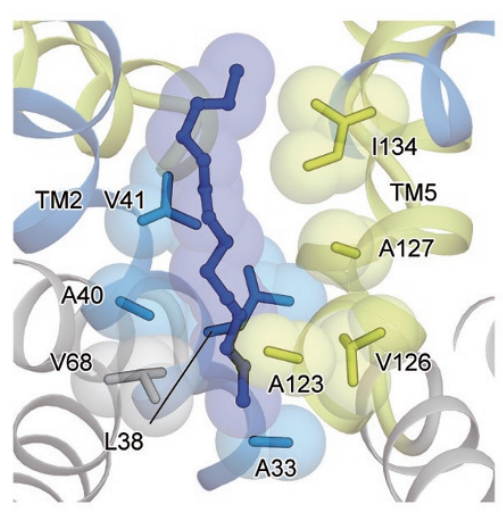

Figure 5 Lateral fenestration. (A) Surface representation of RsTRIC. The TM2 and TM5 helices from two subunits, and the lipid molecule are shown and colored light blue, yellow and blue, respectively. (B) Cross-section of a surface representation of RsTRIC, showing the lateral fenestrations viewed from the periplasmic side. The $F_{\mathrm{o}}-F_{\mathrm{c}}$ electron density map for the carbon acyl chains of the lipid molecules, contoured at $2.5 \sigma$. (C) An enlarged view of the carbon acyl chain of the lipid molecule. (D, E) Close-up views of the lateral fenestrations. The side chains of the residues lining the fenestrations are shown by sphere and stick models. 
A
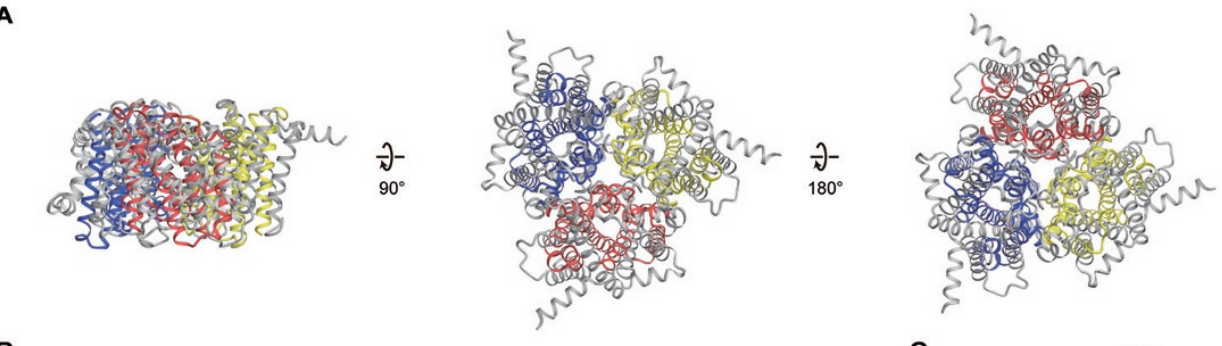

B
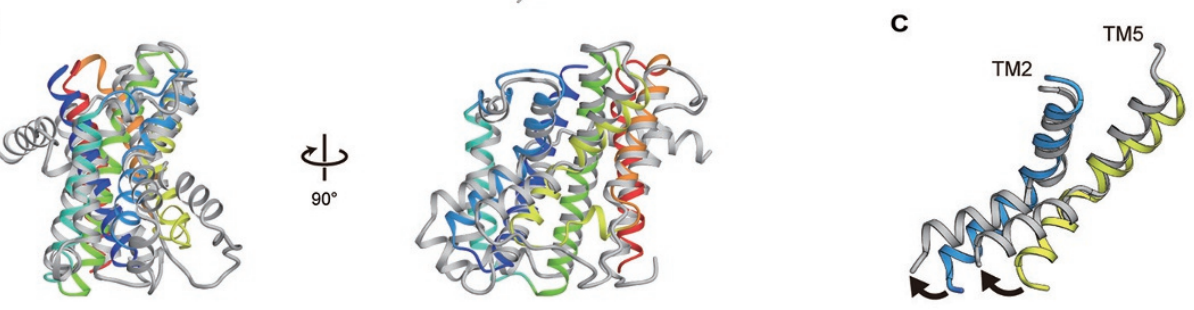

D
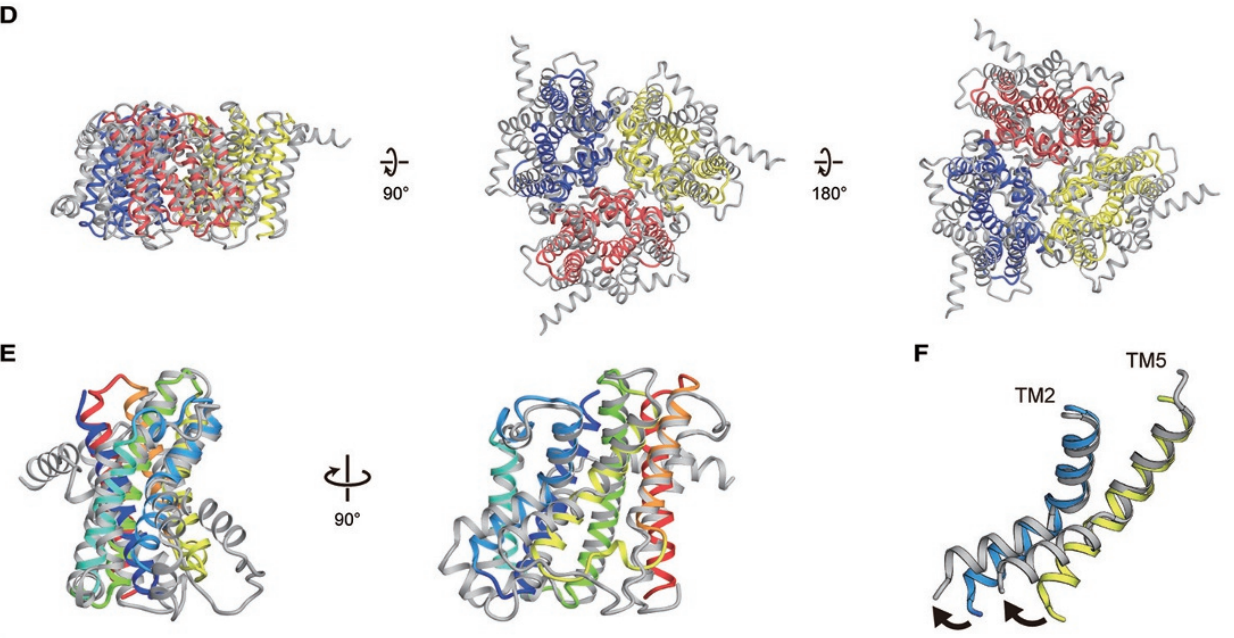

G

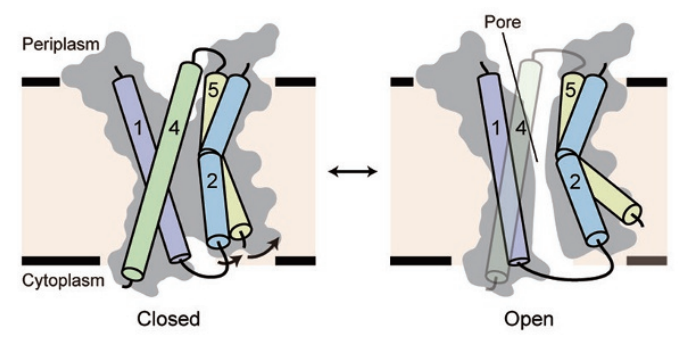

Figure 6 Structural comparisons between prokaryotic and eukaryotic TRIC channels. (A) Representative superimposition of the trimers of SsTRIC and CeTRIC-B2 (PDB ID 5EIK), viewed parallel to the membrane (left), and from the periplasmic (middle) and cytoplasmic (right) sides. SsTRIC is colored as in Figure 2A, and CeTRIC-B2 is colored grey. The RMSD value is $4.5 \AA$ for $461 \mathrm{Ca}$ atoms. (B) Representative superimposition of the SsTRIC subunit and the CeTRIC-B2 subunit, viewed parallel to the membrane. SsTRIC is colored as in Figure 2C, and ceTRIC-B2 is colored grey. The RMSD value is $3.3 \AA$ for $160 \mathrm{C} \alpha$ atoms. (C) Close-up view of the superimposition of the SsTRIC subunit and the CeTRIC-B2 subunit. Only TM2 and TM5 are shown. The black arrows denote the movement of the TM helices. (D) Representative superimposition of the trimers of RsTRIC and CeTRIC-B2, viewed parallel to the membrane (left), and from the periplasmic (middle) and cytoplasmic (right) sides. RsTRIC is colored as in Figure 2B, and CeTRIC-B2 is colored grey. The RMSD value is $4.4 \AA$ for $493 \mathrm{C} \alpha$ atoms. (E) Representative superimposition of the RsTRIC subunit and the CeTRIC-B2 subunit, viewed parallel to the membrane. RsTRIC is colored in the same manner as SsTRIC in Figure 2C, and CeTRIC-B2 is colored grey. The RMSD value is $3.3 \AA$ for $162 \mathrm{C} \alpha$ atoms. (F) Close-up view of the superimposition of the RsTRIC subunit and the CeTRIC-B2 subunit. Only TM2 and TM5 are shown. The black arrows denote the movement of the TM helices. (G) Proposed mechanism of monomeric pore opening of TRIC channels, colored as in Figure $2 \mathrm{C}$. Arrows indicate the kink motions of the TM2 and TM5 helices. TRIC subunits in the closed and open states are shown. 
the CeTRIC-B2 structure solved at higher resolution for the structural comparisons between prokaryotic and eukaryotic TRIC channels (Figure 6). Despite the very low amino-acid sequence identities between the prokaryotic and eukaryotic TRIC channels $(10.9 \%$ between RsTRIC and CeTRIC-B2, and $15.2 \%$ between SsTRIC and CeTRIC-B2), the subunit topology and trimer formation of the prokaryotic TRIC channels are consistent with those of CeTRIC-B2 (Figure 6A-6B and 6D-6E), with relatively high RMSD values (for the details of the RMSD values, see the figure legend of Figure 6). These structural comparisons together with the electrophysiological analyses of RsTRIC and CeTRIC-B revealed the common features such as the monomeric pore [45] (Figures 3,4 and $6 \mathrm{~A}-6 \mathrm{~F}$ ), indicating the mechanistic similarities between the prokaryotic and eukaryotic TRIC channels. Intriguingly, the previously reported electrophysiological analyses of mammalian TRIC channels did not indicate the existence of three monomeric pores within a trimer $[15,22]$, which might, if real, suggest the unique feature of mammalian TRIC channels.

The structural comparisons also revealed the details about their structural differences (Figure 6). First, while both the prokaryotic and eukaryotic TRIC channel structures unveiled lipid binding to the TRIC channels, their binding manners are quite different. In the prokaryotic TRIC channel structures, there are three long and straight lateral fenestrations mainly consisting of the TM2 and TM5 helices, with one end toward the center of the trimer and the other end toward the membrane (Figure 5A and $5 \mathrm{~B}$ ). These lateral fenestrations can accommodate the lipid molecules by providing access from the membrane (Figure 5 and Supplementary information, Figure S7D and S7E). In contrast, there are no such lateral fenestrations in the eukaryotic TRIC channel structures [45]. In the CeTRIC-B structures, while the acyl chains of PIP2 face toward the center of the trimer, the PIP2 head group is oriented toward the monomeric pore at the cytoplasmic side, rather than the lipid bilayer [45]. Notably, the KEVXRXXK consensus sequence motif involved in PIP2 binding is located between the TM5 and TM6 helices, and is highly conserved among eukaryotic TRIC channels, but not among prokaryotic TRIC channels (Supplementary information, Figure S3).

Further structural comparisons revealed the differences in the TM2 and TM5 helices (Figure 6C and 6F). In the CeTRIC-B2 structure, the TM2 and TM5 helices are more kinked at the cytoplasmic side, as compared with those in the prokaryotic TRIC channel structures (Figure $6 \mathrm{C}$ and $6 \mathrm{~F}$ ). Consequently, whereas the monomeric pore is closed on the cytoplasmic side in the prokaryotic TRIC channel structures (Figure 4A and 4C), the cytoplasmic side of the monomeric pore is solvent-accessible in the eukaryotic TRIC channel structures [45]. Thus, the structural differences in the TM2 and TM5 helices might represent the early structural changes in channel activation. Overall, together with the functional analyses of prokaryotic and eukaryotic TRIC channels (Figure 4E, 4F and Supplementary information Figure S6), these structural differences in the kinked TM2 and TM5 helices might further support our hypothesis that the kink motions of the TM2 and TM5 helices may lead to the channel opening (Figure 6G).

\section{Discussion}

The crystal structures of the prokaryotic TRIC channels revealed the architecture of each subunit, with two inverted repeat regions, and the trimerization manner (Figure 2). The electrophysiological analysis of the monomeric mutant showed the monomeric cation-conducting pore of the TRIC channels (Figure 3). The symmetry-related TM2 and TM5 helices are kinked at the conserved glycine clusters that are essential for the ion channel activity (Figures 2, 3 and Supplementary information, Figure S3), and the structure-based functional analysis of eukaryotic TRIC channels demonstrated the functional and mechanistic similarities between the prokaryotic and eukaryotic TRIC channels [42-44] (Supplementary information, Figure S6). Furthermore, we identified lipids bound to the lateral fenestrations at the subunit interfaces, which might modulate the function of the TRIC channels, as observed in other ion channels (Figure 5 and Supplementary information, Figure S7). Intriguingly, the kinked TM2 and TM5 helices contribute to all of the key aspects of the TRIC channels: the trimerization, the pore opening and the generation of lateral fenestrations (Figures 2G, 3, 4 and 5). Finally, the structural comparisons between prokaryotic and eukaryotic TRIC channels revealed the similarities and differences between the distantly related TRIC orthologues, and provided insights into the channel gating (Figure 6).

Taken together, this study presents the structural framework for understanding the molecular mechanisms of this ion channel superfamily, with eukaryotic physiological functions implicated in the $\mathrm{Ca}^{2+}$ homeostasis of the SR and ER.

\section{Materials and Methods}

\section{Expression and purification}

The GFP-tagged RsTRIC $\Delta \mathrm{C} 8$ from $R$. sphaeroides and SsTRIC $\Delta C 7$ from $S$. solfataricus were overexpressed in $E$. coli C41 (DE3) and Rosetta2 (DE3), respectively. Membrane fractions of RsTRIC and SsTRIC were solubilized and purified by chroma- 
tography on a Ni-NTA Superflow column (Qiagen). After dialysis and Tev digestion to remove the GFP-tag, the samples were further purified on Ni-NTA Superflow and Superdex 200 Increase 10/300 GL (GE Healthcare) columns. The SeMet-labeled RsTRIC (RsTRIC $\Delta$ C8, L52M) protein was expressed in E. coli B834 (DE3; Novagen) and purified using the same protocol as that for the native RsTRIC protein. Details of protein expression and purification are provided in the Supplementary information, Data S1.

\section{Preparation of the SsTRIC-Fab complex}

The Fab fragment of a monoclonal antibody against SsTRIC $\Delta C 7$ was prepared in a similar manner as described previously [46]. For crystallization, SsTRIC $\Delta \mathrm{C} 7$ and the Fab fragment were mixed in a molar ratio of 1:2. Details are provided in the Supplementary information, Data S1.

\section{Crystallization}

The crystals of the RsTRIC and SsTRIC proteins were obtained by the sitting drop vapor diffusion method at $20{ }^{\circ} \mathrm{C}$. Details are provided in the Supplementary information, Data S1.

\section{Data collection and structure determination}

X-ray diffraction data sets of RsTRIC and SsTRIC were collected at the SPring-8 beamline BL41XU and the Swiss Light Source beamline X06SA. Data collection and refinement statistics are summarized in the Supplementary information, Table S1. The molecular graphics were illustrated with CueMol (http://www.cuemol.org/). Details for the data collection, structure determination and model refinement are provided in the Supplementary information, Data S1.

\section{In vivo complementation assays of prokaryotic TRIC pro- teins}

To test the $\mathrm{K}^{+}$uptake activity of the prokaryotic TRIC proteins, $\mathrm{K}^{+}$-requiring E. coli strain transformants of RsTRIC or SsTRIC were spotted and streaked on low-potassium medium plates. The concentrations of $\mathrm{KCl}$ and IPTG are indicated in Figure 1B. Details are provided in the Supplementary information, Data S1.

\section{Patch-clamp analysis}

The patch-clamp analysis of the GFP-tagged RsTRIC $\Delta \mathrm{C} 8$ and its mutants using E. coli spheroplasts was performed in the inside-out configuration as described previously [47-49], with minor modifications. For stable giga seal formation, $20 \mathrm{mM} \mathrm{CaCl}_{2}$ in the pipette and bath solutions is required [49]. In Figure $1 \mathrm{E}$ and $3 \mathrm{H}$, single-channel conductances were analyzed from the current-voltage relationships. In Figure 3I, since the single-channel currents were recorded as multiple events, the apparent open probability (Po) was calculated from the mean integrated value of the current amplitude. Details are provided in the Supplementary information, Data S1.

\section{Chemical cross-linking}

The GFP-tagged RsTRIC $\Delta \mathrm{C} 8$ and its mutants were overeproduced in E. coli Rosetta2 (DE3) cells. DSS was added to the solubilized and membrane samples. After incubation at room temperature for $30 \mathrm{~min}$, the products were fractionated by SDS-PAGE and detected by western blot analysis, using an anti-GFP antibody (Nacalai Tesque). Details are provided in the Supplementary infor- mation, Data S1.

\section{Zero-mode waveguides}

RsTRIC with a HaloTag attached to the C-terminus was fluorescently labeled with TMR-HaloLigand (Promega) by an incubation for $2 \mathrm{~h}$ on ice. Nanodiscs were formed by mixing the TMR-RsTRIC channel, BCCP (Biotin Carboxyl Carrier Protein)-MSP1E3 and lipids derived from an E. coli soluble fraction, as described previously $[50,51]$. Each TMR-TRIC channel incorporated into nanodiscs was immobilized at the bottom of the ZMWs surface through a biotin-avidin bond (SMRT Cell, Pacific Bioscience, Inc.). The commercial ZMW surface was modified with PEG and biotin-PEG. For statistical analysis, multiple traces ( $n=100$ in total) were analyzed by counting the number of photobleaching steps, as described previously [52]. Figure 3F shows the TMR-labeling efficiencies for RsTRIC $\Delta \mathrm{C} 8$ and the monomeric mutant.

\section{Thermostability analysis by FSEC-TS}

The thermostability analysis by FSEC-TS was performed as described previously [33]. In brief, the peak heights of RsTRIC samples incubated at $30{ }^{\circ} \mathrm{C}-90{ }^{\circ} \mathrm{C}$ were normalized to that of the sample incubated at $4{ }^{\circ} \mathrm{C}$, and were fit to the Hill equation. The melting temperatures $\left(T_{\mathrm{m}}\right)$ were determined by fitting the curves to a sigmoidal equation.

\section{$\mathrm{KCl}$ tolerance assay in yeasts}

The hyper-potassium salt-sensitive strain Y291 (BY4742

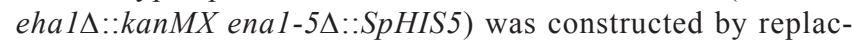
ing the cluster of three ena homologous genes, ENA1, ENA2 and ENA5, hereafter denoted as enal-ena5, in the BY4742 eha1 $:$ ::kanMX strain [53] (Open Biosystems), using a DNA fragment containing the SpHIS5 marker gene as the PCR template and the pair of knockout primers ENA-A/ENA-B and pUG27 [54]. Since the parental BY4742 strain, a derivative of S288C, harbors three tandem copies of ENA genes, while the previously reported parental strain W303-1A contains four [40], the BY4742 strain exhibits intrinsically weak potassium salt sensitivity [55]. The expression screening of eukaryotic TRIC channels by FSEC identified ceTRIC-1 as a promising candidate for functional analysis in yeast. ceTRIC-1 was subcloned into the $\mathrm{p} 416 \mathrm{GPD}$ vector, with the alpha-mating factor signal sequence at the N-terminus and a GFPHis8 tag following an ER export signal [41] at the C-terminus. The putatively disordered $24 \mathrm{C}$-terminal residues of ceTRIC-1 were truncated, as in the truncated constructs of RsTRIC and SsTRIC. URA+ transformants harboring ceTRIC-1 plasmids were obtained on SC-URA plates. Each transformant colony was picked, spotted onto a set of plates with different $\mathrm{KCl}$ concentrations as indicated in the Supplementary information, Figure S6A and streaked. The plates were incubated for 6 days at $30{ }^{\circ} \mathrm{C}$.

\section{Microscopic analysis}

Fluorescence imaging of yeast cells was performed with a microscope (Carl Zeiss ELYRA PS.1). Yeast cells were grown in SCURA medium to mid-log phase $\left(\mathrm{OD}_{600}\right.$ of 0.6$)$ and then fixed with cold methanol. Fixed cells were washed with PEMS buffer and suspended in fresh buffer for imaging. Fluorescence was detected with a $63 \times$ Plan-Apochromat Objective, with excitation at $488 \mathrm{~nm}$. SIM images were collected at $400 \mathrm{~nm} z$ axis steps, with three rota- 
tions of the structured illumination grid. The resulting stacks were processed by the ZEN 2011 software, using the default reconstruction parameters. Acquired images were processed with Photoshop (Adobe).

\section{Accession codes}

The atomic coordinates and structure factors for RsTRIC and SsTRIC have been deposited in the Protein Data Bank, under the accession codes $5 \mathrm{H} 36$ and $5 \mathrm{H} 35$, respectively.

\section{Acknowledgments}

We thank the Nureki lab members, especially Nishizawa $\mathrm{T}$ and Nishimasu $\mathrm{H}$, for critical comments on the manuscript and $\mathrm{Ku}$ rabayashi A for technical assistance. We thank Watanabe Y (The University of Tokyo) for providing the microscope, the RIKEN BioResource Center for providing $R$. sphaeroides and $S$. solfataricus genomic DNAs, and the beam-line staffs at BL41XU and BL32XU of SPring-8, PX I and PX II of the Swiss Light Source and BL1A of the Photon Factory for assistance in data collection. The diffraction experiments were performed at SPring-8 BL41XU and BL32XU (proposals 2014A1091, 2014A1061, 2014A1093, 2014A1116 and 2014B1194) and the Swiss Light Source. This work was supported by the Platform for Drug Discovery, Informatics and Structural Life Science of the Ministry of Education, Culture, Sports, Science and Technology (MEXT), JSPS KAKENHI (22117007, 24227004, 25291011 and 26711003), the FIRST program, PRESTO, JST grants to Mo H, the National Natural Science Foundation of China ( 31570838 to Mo H), the Ministry of Science and Technology of China (2016YFA0502800 to Mo H), the National Thousand Young Talents award from the Office of Global Experts Recruitment in China to Mo H, CREST, JST grants to TK and SU, a Grant-in-Aid for JSPS Fellows, grants for the HPCI STRATEGIC PROGRAM Computational Life Science and Application in Drug Discovery and Medical Development, a Grantin-Aid for Scientific Research on Innovative Areas ('Non-coding RNA neo-taxonomy') from MEXT, and grants from the Mochida Memorial Foundation and the Nakajima Foundation to Mo $\mathrm{H}$.

\section{Author contributions}

$\mathrm{MoH}$ and $\mathrm{ON}$ conceived the project. SI, SU, KI, RI, MoH and ON designed the experiments. HEK provided help for the experiments. GK, MaH, ADM, KL, YNN, KT, TK, YG, TN, MT, MW and KI performed the experiments. GK, MaH, ADM, KK, YF, KL, YNN, KT, TK, SU, YG, TN, MT, KY, MW, KI, RI and MoH analyzed the data. GK, $\mathrm{MoH}$ and $\mathrm{ON}$ wrote the manuscript.

\section{Competing Financial Interests}

The authors declare no competing financial interests.

\section{References}

1 Berridge MJ, Bootman MD, Roderick HL. Calcium signalling: dynamics, homeostasis and remodelling. Nat Rev Mol Cell Biol 2003; 4:517-529.

2 Berridge MJ. Calcium microdomains: organization and function. Cell Calcium 2006; 40:405-412.

3 Stutzmann GE, Mattson MP. Endoplasmic reticulum $\mathrm{Ca}^{2+}$ handling in excitable cells in health and disease. Pharmacol Rev 2011; 63:700-727.

4 Bers DM. Cardiac sarcoplasmic reticulum calcium leak: basis and roles in cardiac dysfunction. Ann Rev Physiol 2014; 76:107-127.

5 Parys JB, De Smedt H. Inositol 1,4,5-trisphosphate and its receptors. Adv Exp Med Biol 2012; 740:255-279.

6 Brini M, Carafoli E. Calcium pumps in health and disease. Physiol Rev 2009; 89:1341-1378.

7 Toyoshima C. Structural aspects of ion pumping by $\mathrm{Ca}^{2+}$-ATPase of sarcoplasmic reticulum. Arch Biochem Biophys 2008; 476:3-11.

8 Fan G, Baker ML, Wang Z, et al. Gating machinery of InsP3R channels revealed by electron cryomicroscopy. Nature 2015; 527:336-341.

9 Ludtke SJ, Tran TP, Ngo QT, Moiseenkova-Bell VY, Chiu W, Serysheva, II. Flexible architecture of IP3R1 by Cryo-EM. Structure 2011; 19:1192-1199.

10 Yan Z, Bai XC, Yan C, et al. Structure of the rabbit ryanodine receptor RyR1 at near-atomic resolution. Nature 2015; 517:50-55.

11 Bosanac I, Alattia JR, Mal TK, et al. Structure of the inositol 1,4,5-trisphosphate receptor binding core in complex with its ligand. Nature 2002; 420:696-700.

12 Coronado R, Miller C. Decamethonium and hexamethonium block $\mathrm{K}^{+}$channels of sarcoplasmic reticulum. Nature 1980; 288:495-497.

13 Fink RH, Veigel C. Calcium uptake and release modulated by counter-ion conductances in the sarcoplasmic reticulum of skeletal muscle. Acta Physiol Scand 1996; 156:387-396.

14 Meissner G. Monovalent ion and calcium ion fluxes in sarcoplasmic reticulum. Mol Cell Biochem 1983; 55:65-82.

15 Yazawa M, Ferrante C, Feng J, et al. TRIC channels are essential for $\mathrm{Ca}^{2+}$ handling in intracellular stores. Nature 2007; 448:78-82.

16 Guo T, Nani A, Shonts S, et al. Sarcoplasmic reticulum $\mathrm{K}^{+}$ (TRIC) channel does not carry essential countercurrent during $\mathrm{Ca}^{2+}$ release. Biophys J 2013; 105:1151-1160.

17 Silverio AL, Saier MH, Jr. Bioinformatic characterization of the trimeric intracellular cation-specific channel protein family. J Membr Biol 2011; 241:77-101.

18 Venturi E, Sitsapesan R, Yamazaki D, Takeshima H. TRIC channels supporting efficient $\mathrm{Ca}^{2+}$ release from intracellular stores. Pflugers Arch 2013; 465:187-195.

19 Zhou X, Lin P, Yamazaki D, et al. Trimeric intracellular cation channels and sarcoplasmic/endoplasmic reticulum calcium homeostasis. Circ Res 2014; 114:706-716.

20 Takeshima H, Venturi E, Sitsapesan R. New and notable ion-channels in the sarcoplasmic/endoplasmic reticulum: do they support the process of intracellular $\mathrm{Ca}^{2+}$ release? J Physiol 2014.

21 Kuum M, Veksler V, Kaasik A. Potassium fluxes across the endoplasmic reticulum and their role in endoplasmic reticulum calcium homeostasis. Cell Calcium 2014.

22 Pitt SJ, Park KH, Nishi M, et al. Charade of the SR K ${ }^{+}$-channel: two ion-channels, TRIC-A and TRIC-B, masquerade as a single $\mathrm{K}^{+}$-channel. Biophys $J$ 2010; 99:417-426.

23 Venturi E, Matyjaszkiewicz A, Pitt SJ, et al. TRIC-B channels display labile gating: evidence from the TRIC-A knockout 
mouse model. Pflugers Arch 2013; 465:1135-1148.

24 Yamazaki D, Tabara Y, Kita S, et al. TRIC-A channels in vascular smooth muscle contribute to blood pressure maintenance. Cell Metab 2011; 14:231-241.

25 Zhao X, Yamazaki D, Park KH, et al. $\mathrm{Ca}^{2+}$ overload and sarcoplasmic reticulum instability in tric a null skeletal muscle. The J Biol Chem 2010; 285:37370-37376.

26 Tao S, Yamazaki D, Komazaki S, et al. Facilitated hyperpolarization signaling in vascular smooth muscle-overexpressing TRIC-A channels. J Biol Chem 2013; 288:15581-15589.

27 Yamazaki D, Komazaki S, Nakanishi H, et al. Essential role of the TRIC-B channel in $\mathrm{Ca}^{2+}$ handling of alveolar epithelial cells and in perinatal lung maturation. Development 2009; 136:2355-2361.

28 Zhao C, Ichimura A, Qian N, et al. Mice lacking the intracellular cation channel TRIC-B have compromised collagen production and impaired bone mineralization. Science signaling 2016; 9:ra49.

29 Shaheen R, Alazami AM, Alshammari MJ, et al. Study of autosomal recessive osteogenesis imperfecta in Arabia reveals a novel locus defined by TMEM38B mutation. J Med Genet 2012; 49:630-635.

30 Rubinato E, Morgan A, D'Eustacchio A, et al. A novel deletion mutation involving TMEM38B in a patient with autosomal recessive osteogenesis imperfecta. Gene 2014; 545:290292.

31 Volodarsky M, Markus B, Cohen I, et al. A deletion mutation in TMEM38B associated with autosomal recessive osteogenesis imperfecta. Hum Mutat 2013; 34:582-586.

32 Kawate T, Gouaux E. Fluorescence-detection size-exclusion chromatography for precrystallization screening of integral membrane proteins. Structure 2006; 14:673-681.

33 Hattori M, Hibbs RE, Gouaux E. A fluorescence-detection size-exclusion chromatography-based thermostability assay for membrane protein precrystallization screening. Structure 2012; 20:1293-1299.

34 Altendorf K, Epstein W, Lohmann A. Valinomycin-induced cation transport in vesicles does not reflect the activity of $\mathrm{K}^{+}$transport systems in Escherichia coli. J Bacteriol 1986; 166:334-337.

35 von Heijne G. Membrane protein structure prediction. Hydrophobicity analysis and the positive-inside rule. $\mathrm{J} \mathrm{Mol} \mathrm{Biol}$ 1992; 225:487-494.

36 Feilmeier BJ, Iseminger G, Schroeder D, Webber H, Phillips GJ. Green fluorescent protein functions as a reporter for protein localization in Escherichia coli. J Bacteriol 2000; 182:4068-4076.

37 Levene MJ, Korlach J, Turner SW, Foquet M, Craighead HG, Webb WW. Zero-mode waveguides for single-molecule analysis at high concentrations. Science 2003; 299:682-686.

38 Zhu P, Craighead HG. Zero-mode waveguides for single-molecule analysis. Ann Rev Biophys 2012; 41:269-293.

39 Uemura S, Aitken CE, Korlach J, Flusberg BA, Turner SW, Puglisi JD. Real-time tRNA transit on single translating ribosomes at codon resolution. Nature 2010; 464:1012-1017.

40 Arino J, Ramos J, Sychrova H. Alkali metal cation transport and homeostasis in yeasts. Microbiol Mol Biol Rev 2010; 74:95-120.

41 Votsmeier C, Gallwitz D. An acidic sequence of a putative yeast Golgi membrane protein binds COPII and facilitates ER export. EMBO J 2001; 20:6742-6750.

42 Payandeh J, Scheuer T, Zheng N, Catterall WA. The crystal structure of a voltage-gated sodium channel. Nature 2011; 475:353-358.

43 Zhang X, Ren W, DeCaen P, et al. Crystal structure of an orthologue of the $\mathrm{NaChBac}$ voltage-gated sodium channel. Nature 2012; 486:130-134.

44 Brohawn SG, Campbell EB, MacKinnon R. Physical mechanism for gating and mechanosensitivity of the human TRAAK $\mathrm{K}^{+}$channel. Nature 2014; 516:126-130.

45 Yang H, Hu M, Guo J, Ou X, Cai T, Liu Z. Pore architecture of TRIC channels and insights into their gating mechanism. Nature 2016.

46 Hino T, Arakawa T, Iwanari H, et al. G-protein-coupled receptor inactivation by an allosteric inverse-agonist antibody. Nature 2012; 482:237-240.

47 Martinac B, Buechner M, Delcour AH, Adler J, Kung C. Pressure-sensitive ion channel in Escherichia coli. Proc Natl Acad Sci USA 1987; 84:2297-2301.

48 Hattori M, Iwase $\mathrm{N}$, Furuya $\mathrm{N}$, et al. $\mathrm{Mg}^{2+}$-dependent gating of bacterial MgtE channel underlies $\mathrm{Mg}^{2+}$ homeostasis. EMBO $J$ 2009; 28:3602-3612.

49 Kuo MM, Saimi Y, Kung C, Choe S. Patch clamp and phenotypic analyses of a prokaryotic cyclic nucleotide-gated $\mathrm{K}^{+}$ channel using Escherichia coli as a host. J Biol Chem 2007; 282:24294-24301.

50 Bayburt TH, Grinkova YV, Sligar SG. Self-assembly of discoidal phospholipid bilayer nanoparticles with membrane scaffold proteins. Nano Lett 2002; 2:853-856.

51 Denisov IG, Grinkova YV, Lazarides AA, Sligar SG. Directed self-assembly of monodisperse phospholipid bilayer nanodiscs with controlled Size. J Am Chem Soc 2004; 126:34773487.

52 Ulbrich MH, Isacoff EY. Subunit counting in membrane-bound proteins. Nat Methods 2007; 4:319-321.

53 Winzeler EA, Shoemaker DD, Astromoff A, et al. Functional characterization of the S. cerevisiae genome by gene deletion and parallel analysis. Science 1999; 285:901-906.

54 Gueldener U, Heinisch J, Koehler GJ, Voss D, Hegemann JH. A second set of loxP marker cassettes for Cre-mediated multiple gene knockouts in budding yeast. Nucleic Acids Res 2002; 30:e23.

55 Petrezselyova S, Zahradka J, Sychrova H. Saccharomyces cerevisiae BY4741 and W303-1A laboratory strains differ in salt tolerance. Fungal Biol 2010; 114:144-150.

(Supplementary information is linked to the online version of the paper on the Cell Research website.)

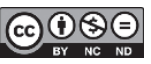

This work is licensed under a Creative Commons Attribution-NonCommercial-NoDerivs 4.0 Unported License. The images or other third party material in this article are included in the article's Creative Commons license, unless indicated otherwise in the credit line; if the material is not included under the Creative Commons license, users will need to obtain permission from the license holder to reproduce the material. To view a copy of this license, visit http://creativecommons.org/licenses/by-nc-nd/4.0/

(C) The Author(s) 2016 